\title{
Endpoint Formulas for Interpolatory Cubic Splines*
}

\author{
By Thomas I. Seidman and Robert J. Korsan
}

\begin{abstract}
In the absence of known endpoint derivatives, the usual procedure is to use a "natural" spline interpolant which Kershaw has shown to have $O\left(h^{4}\right)$ error except near the endpoints. This note observes that either the use of appropriate finite-difference approximations for the endpoint derivatives or a proposed modification of the interpolation algorithm leads to $\mathcal{O}\left(h^{4}\right)$ error uniformly in the interval of approximation.
\end{abstract}

We consider the interpolation to a function $x \in C^{4}[a, b]$ by cubic splines. Let $\left\{a=t_{0}, t_{1}, \cdots, t_{n}=b\right\}$ be a set of knots and, throughout, let $y$ denote a cubic spline interpolant to $x$ so that $y\left(t_{i}\right)=x_{i}=x\left(t_{i}\right)$ for $j=0, \cdots, N$. The construction of $y$ may be given in terms of the values $\left\{x_{j}\right\}$ and $\left\{\kappa_{j}\right\}$ with $\kappa_{j}=y^{\prime \prime}\left(t_{j}\right)$ for $j=0, \cdots, N$. Kershaw [1] provides the estimates

$$
\left|x^{(k)}(t)-y^{(k)}(t)\right| \leqq c_{k} h^{2-k}\left[h^{2} M+8 \max _{j}\left\{\left|x^{\prime \prime}\left(t_{j}\right)-\kappa_{j}\right|\right\}\right],
$$

for $a \leqq t \leqq b$ and $k=0,1,2$; here, $h=\max _{j}\left\{t_{j+1}-t_{j}\right\}$ and $M=$ $\sup \left\{\left|x^{(4)}(t)\right|: a \leqq t \leqq b\right\}$. For $y$ to be a cubic spline, the values $\left\{\kappa_{j}\right\}$ must satisfy

$$
\alpha_{j} \kappa_{i-1}+2 \kappa_{j}+\left(1-\alpha_{i}\right) \kappa_{j+1}=6 d_{i}, \quad j=1, \cdots, N-1,
$$

where

$$
\begin{aligned}
& h_{i}=t_{i+1}-t_{i}, \quad \alpha_{i}=h_{j-1} /\left(h_{i-1}+h_{j}\right), \\
& d_{i}=\left[\left(1-\alpha_{j}\right) x_{i-1}-x_{i}+\alpha_{j} x_{i+1}\right] / h_{j-1} h_{j} .
\end{aligned}
$$

Kershaw has shown [1] that the Eqs. (2), together with either

$$
y^{\prime}(a)=x^{\prime}(a), \quad y^{\prime}(b)=x^{\prime}(b)
$$

(giving the $D-1$ spline interpolant) or

$$
\kappa_{0}=x^{\prime \prime}(a), \quad k_{N}=x^{\prime \prime}(b)
$$

(giving the $D-2$ spline interpolant), are sufficient to determine all the values $\left\{\kappa_{0}, \cdots, \kappa_{N}\right\}$, and hence $y$, in such a way that

$$
\max _{j}\left\{\left|x^{\prime \prime}\left(t_{i}\right)-\kappa_{i}\right|\right\}=\mathcal{O}\left(h^{2} M\right)
$$

Received November 15, 1971.

AMS 1970 subject classifications. Primary 41A05, 41A15, 41A25; Secondary 65D15.

Key words and phrases. Interpolation, cubic splines, endpoint formula.

* The results reported in this paper were obtained while the senior author (T.I.S.) was at Carnegie-Mellon University and the junior author (R.J.K.) was at the Westinghouse Research Laboratories. Acknowledgement and thanks are due to the Westinghouse Research Laboratories for their support.

Copyright @ 1972, American Mathematical Society 
which, with (1), gives the estimates

$$
\left|x^{(k)}(t)-y^{(k)}(t)\right| \leqq \mathcal{O}\left(h^{4-k} M\right), \quad k=0,1,2,
$$

uniformly on $[a, b]$.

The standard practice in the absence of information about $x^{\prime}(a), x^{\prime}(b)$ or $x^{\prime \prime}(a)$, $x^{\prime \prime}(b)$ is to use the "natural spline" interpolant, using (2) together with

$$
\kappa_{0}=0, \quad \kappa_{N}=0,
$$

to determine the $\left\{\kappa_{j}\right\}$ and therefore $y$. In this case, it is shown in [1] that the estimate (5) still holds for $t$ in the interior of the interval but that the error is of lower order, in general, for $t$ within $\mathcal{O}(h \log h)$ of the endpoints. It is our present intention to provide two methods for retaining the estimate (5) uniformly on $[a, b]$ without $a$ priori knowledge of endpoint derivatives.

Observe, first, that (3.1) is equivalent, in conjunction with the system (2) to the pair of equations

$$
2 \kappa_{0}+\kappa_{1}=6 d_{0}, \quad \kappa_{N-1}+2 \kappa_{N}=6 d_{N}
$$

with

$$
\begin{aligned}
& d_{0}=\left[x_{1}-x_{0}-h_{0} p\right] / h_{0}^{2}, \\
& d_{N}=-\left[x_{N}-x_{N-1}-h_{N-1} q\right] / h_{N-1}^{2},
\end{aligned}
$$

where $p=x^{\prime}(a)$ and $q=x^{\prime}(b)$. The combined system (2) $+(6)$ can be written in the form $A \boldsymbol{x}=\mathrm{d}$ with

$$
\mathbf{A}=\left(\begin{array}{cccccc}
2 & 1 & 0 & \cdots & 0 \\
\alpha_{1} & 2 & 1-\alpha_{1} & 0 & 0 \\
0 & \alpha_{2} & 2 & 1-\alpha_{2} & \vdots \\
\vdots & \ddots & \ddots & \ddots & \ddots & \\
0 & \cdots & & 0 & 1 & 2
\end{array}\right), \quad \boldsymbol{\kappa}=\left(\begin{array}{c}
\kappa_{0} \\
\vdots \\
\kappa_{N}
\end{array}\right), \quad \mathbf{d}=\left(\begin{array}{c}
d_{0} \\
\vdots \\
d_{N}
\end{array}\right)
$$

and we note that $\left\|\frac{1}{2} \mathrm{~A}-\mathrm{I}\right\|_{\infty}=\frac{1}{2}$, so that

$$
\left\|\mathbf{A}^{-1}\right\|_{\infty}=\frac{1}{2}\left\|\left(\frac{1}{2} \mathbf{A}\right)^{-1}\right\| \leqq \frac{1}{2} /\left[1-\left\|\frac{1}{2} \mathbf{A}-\mathbf{I}\right\|_{\infty}\right]=1 \text {. }
$$

It follows that if we replace $p$ by $p_{*}$ and $q$ by $q_{*}$ in (7), leaving the vector $\mathrm{d}$ otherwise unchanged, no component of $\boldsymbol{k}$ is altered by more than

$$
6|| \mathbf{A}^{-1}\left\|_{\infty}\right\| d-d_{*} \| \leqq 6 \max \left\{\left|p-p_{*}\right| / h_{0},\left|q-q_{*}\right| / h_{N-1}\right\} .
$$

Thus, the estimate (4) continues to hold-and so (5) holds uniformly on $[a, b]-$ if we take $p_{*}, q_{*}$ to be approximations to $p, q$ with accuracy $\mathcal{O}\left(h^{3} M\right)$. This can be done using appropriate four-point difference formulas; to be precise, we may define $p_{*}$ by

$$
p_{*}=a_{0} x_{0}+a_{1} x_{1}+a_{2} x_{2}+a_{3} x_{3},
$$

where 
(9)

$$
\begin{aligned}
& a_{1}=\left(h_{0}+h_{1}\right)\left(h_{0}+h_{1}+h_{2}\right) / h_{0} h_{1}\left(h_{1}+h_{2}\right), \\
& a_{2}=-\left(h_{0}+h_{1}+h_{2}\right) h_{0} / h_{1} h_{2}\left(h_{0}+h_{1}\right), \\
& a_{3}=\left(h_{0}+h_{1}\right) h_{0} /\left(h_{0}+h_{1}+h_{2}\right)\left(h_{1}+h_{2}\right) h_{2}, \\
& a_{0}=-\left(a_{1}+a_{2}+a_{3}\right),
\end{aligned}
$$

and similarly for $q_{*}$.

One could, alternatively, use four-point difference formulas to obtain approximations to $x^{\prime \prime}(a), x^{\prime \prime}(b)$ of accuracy $\mathcal{O}\left(h^{2} M\right)$ for use in (3.2). A simpler method, modifying the system (6) for $j=1, N-1$ rather than directly approximating $\kappa_{0}, \kappa_{N}$, depends on the observation that

$$
\left(1-\alpha_{j}\right) x^{\prime \prime}\left(t_{j-1}\right)-x^{\prime \prime}\left(t_{j}\right)+\alpha_{j} x^{\prime \prime}\left(t_{j+1}\right)=\mathcal{O}\left(h^{2} M\right), \quad j=1, \cdots, N-1,
$$

and, further, that

$$
\begin{aligned}
\alpha_{j} x^{\prime \prime}\left(t_{i-1}\right)+2 x^{\prime \prime}\left(t_{i}\right)+\left(1-\alpha_{j}\right) x^{\prime \prime}\left(t_{i+1}\right)=6 d_{j}+\mathcal{O}\left(h^{2} M\right), & \\
& j=1, \cdots, N-1 .
\end{aligned}
$$

For $j=1$, one may eliminate $x^{\prime \prime}\left(t_{i-1}\right)=x^{\prime \prime}(a)$ between (10) and (11) to obtain

$$
\left(2-\alpha_{1}\right) x^{\prime \prime}\left(t_{1}\right)+\left(1-2 \alpha_{1}\right) x^{\prime \prime}\left(t_{2}\right)=6\left(1-\alpha_{1}\right) d_{1}+\mathcal{O}\left(h^{2} M\right)
$$

and, proceeding similarly for $j=N-1$,

$$
\left(2 \alpha_{N-1}-1\right) x^{\prime \prime}\left(t_{N-2}\right)+\left(1+2 \alpha_{N-1}\right) x^{\prime \prime}\left(t_{N-1}\right)=6 \alpha_{N-1} d_{N-1}+\mathcal{O}\left(h^{2} M\right) .
$$

We may divide (12) by $\left(1-\alpha_{1}\right)$ and (13) by $\alpha_{N-1}$ and then combine these with (11), for $j=2, \cdots, N-2$, to obtain the system $\mathbf{B k}=6 \mathrm{~d}^{\prime}+\mathcal{O}\left(h^{2} M\right)$ where we have set

$$
\mathbf{B}=\left(\begin{array}{ccccc}
2+r & 1-r & 0 & \cdots & 0 \\
\alpha_{2} & 2 & 1-\alpha_{2} & & 0 \\
0 & \ddots & \ddots & \ddots & \vdots \\
\vdots & \ddots & \alpha_{N-2} & 2 & 1-\alpha_{N-2} \\
0 & \cdots & 0 & 1-r^{\prime} & 2+r^{\prime}
\end{array}\right), \mathbf{k}=\left(\begin{array}{c}
x^{\prime \prime}\left(t_{1}\right) \\
\vdots \\
x^{\prime \prime}\left(t_{N-1}\right)
\end{array}\right), \mathbf{d}^{\prime}=\left(\begin{array}{c}
d_{1} \\
\vdots \\
d_{N-1}
\end{array}\right)
$$

with $r=\alpha_{1} /\left(1-\alpha_{1}\right), r^{\prime}=\left(1-\alpha_{N-1}\right) / \alpha_{N-1}$. Note that $\mathrm{B}$ is diagonally dominant and that imposing a bound on $r, r^{\prime}$ imposes a uniform bound (i.e., independent of $N, h)$ on $\left\|\mathbf{B}^{-1}\right\|_{\infty}$. If we obtain $\boldsymbol{k}$ by solving the system $\mathbf{B} \boldsymbol{k}^{\prime}=6 \mathbf{d}^{\prime}\left[\boldsymbol{k}^{\prime}=\left(\kappa_{1}, \cdots, \kappa_{N-1}\right)^{*}\right]$ and subsequently setting

$$
\kappa_{0}=6 d_{1}-\kappa_{1}-\kappa_{2}, \quad \kappa_{n}=6 d_{N-1}-\kappa_{N-1}-\kappa_{N-2}
$$

then the boundedness of $\left\|B^{-1}\right\|_{\infty}$ together with (10), (11) imply the estimate (4) and the estimates (5) follow uniformly on $[a, b]$ from (1).

Observe that if $h_{0}=h_{1}$, then $\alpha_{1}=\frac{1}{2}$ and (12) gives $\kappa_{1}$ by the usual three-point difference formula (accurate to $\mathcal{O}\left(h^{2} M\right)$ with this spacing); similarly for $\kappa_{N-1}$ if $h_{N-2}=$ $h_{N-1}$ so $\alpha_{N-1}=\frac{1}{2}$. In this case the original system (6) can be used for $j=$ $2, \cdots, N-2$ with $\kappa_{1}=2 d_{1}, \kappa_{N-1}=2 d_{N-1}$ and, subsequently, $\kappa_{0}=2 \kappa_{1}-\kappa_{2}$ and 
$\kappa_{N}=2 \kappa_{N-1}-\kappa_{N-2}$. Under normal circumstances, this last would seem to be the method of choice.

University of Maryland, Baltimore County

Baltimore, Maryland 21228

Scientific Time-Sharing Corporation

1420 Avon Place

Pittsburgh, Pennsylvania 15235

1. D. Kershaw, "A note on the convergence of interpolatory cubic splines," SIAM J. Numer. Anal., v. 8, 1971, pp. 67-74. 